\title{
Comparative study for Active Noise Cancellation using Adaptive filtering and Standing wave pattern
}

\author{
Ahmad Abubakar ${ }^{a}$, Isam Janajreh ${ }^{b} *$ \\ ${ }^{a}$ Khalifa University of Science and Technology, Abu-Dhabi, United Arab Emirate \\ ${ }^{b}$ Khalifa University of Science and Technology, Abu-Dhabi, United Arab Emirate
}

\begin{abstract}
Noise pollution is one of the most fundamental challenges facing our environment, causes health problem, communication inefficiency and degrade the performance of works due to lack of concentration, thus, mitigating this impact becomes an unavoidable requirement of time to protect people's health and the environment. This noise may originate from several sources including industrial machinery, system parts wear out, and adjacent environmental acoustics. To mitigate this noise effect, an Active Noise Cancellation (ANC) headphone is achieved by two effective techniques; Adaptive filtering and Standing wave phenomenon. In this work, an ANC system is designed using both adaptive filtering and standing wave techniques, the former one basically utilizes single-channel feedforward whereas the latter one utilizes both single-channel feedforward and feedback control. LMS adaptive filter algorithm is the basic component of the designed ANC headphone. For simulation, a noise-free signal will be used as the desired audio signal and a gaussian distributed noise as the unwanted noise signal, these are combined to form noise corrupted speech signal. Propose algorithms performance were evaluated based on the ability to mitigate effects of different frequency broad-band noise signals and of different Noise to Signal ratio. Evaluation measures used are; convergence rate and noise reduction in $\mathrm{dB}$. Result reveals ANC headphone using standing wave technique has better performance at mitigating noise frequency below $800 \mathrm{~Hz}$, with low SNR than Adaptive filtering. However, at higher frequencies above $1000 \mathrm{~Hz}$, ANC headphone using Adaptive filtering has good performance of masking high frequencies up to $22 \mathrm{~dB}$.
\end{abstract}

Keywords: Active noise cancellation, Adaptive filtering, standing wave pattern, system identification, feedforward and feedback control.

\section{Introduction}

Noise problems are becoming more severe in our society, which has serious negative impacts on our environment [1], as such the need to eliminate them is highly obligatory. The noise problem is an expression of the limited tolerance to the perception of sounds that we have as individuals [2]. Any unwanted audible signal is referred to as Acoustic noise [3], [4]. With the cotemporal growth of technology and industry, acoustic noise levels in human settings are under critical study for reasons including health concerns, communication efficiency and work performance improvement [4]. Most Common acoustic noises are generated by industrial equipment, appliances, and humangenerated noise [4]. Machines, blowers, fans, and transformers are few examples of equipment and appliance mention in [5] that produce signals that are nuisance to the human ear. On the other hand, human voice at a certain sound pressure level is often considered as an unwanted signal, which is an external interruption in communications [6]. 275 million workers around

${ }^{*}$ Corresponding author. Tel.: +1234567890

Fax: +9876543210; E-mail: Author.Name@iasks.org

(C) 2016 International Association for Sharing Knowledge and Sustainability

DOI: $10.5383 /$ ijtee.17.02.001 the world between 50-59 years of age have compensable noiseinduced hearing loss, and it is estimated that $26 \%$ of the working population are employed in jobs with noise exposure higher than $80 \mathrm{~dB}$ [1], [2], [7]. Owing to the concern effect of noise pollution, researchers aimed to explore how to eliminate this noise impact in various application.

According to Sen $\mathrm{M}$ et al. [5], acoustic noise can be classified into two kinds; broadband turbulent or random noise which uniformly distributes its energy over the frequency band and the narrowband or pure tone which concentrate its energy on a specific frequency [8]. Several approaches have been proposed in noise mitigation. For high-frequency noise, classical Passive Noise Cancellation (PCN) methods such as earmuffs, earplugs, audio-limiters use a mechanical barrier to absorb the sound signal, thereby masking a reasonable among of the noise [9]. Wang et al. [10] mitigate the effect of tonal noise by $5 \mathrm{~dB}$ from the forward-curved centrifugal fan analysed in [11] using the open-cell metal foam. However, low-frequency noise cannot be mitigated using passive methods, this is because the width of the mechanical barrier use in passive methods is proportional and very close in value to the noise signal wavelength [9], [12]. 
Passive methods provide a poor response to low-frequency sounds when their wavelength is longer than the size of the muffler liabilities [5]. As such low-frequency noise tend to have a large wavelength, thereby making passive methods for this noise ineffective and very bulky [4], [5]. This lead to what is called the Active Noise Cancellation (ANC) systems such as ANC headphones according to [4], [13]. This system employs the use of electromechanical or electro-acoustic system to reduce the noise effect. ANC was first used in practice back in the 1950s, based on patents by Lawrence Fogel. The two most effective ANC techniques to mitigate low-frequency unwanted sounds in noisy environments are; Adaptive filtering and standing wave pattern, used in [9], [14], [15]. The relevance in the treatment of low-frequency sounds is that they produce very intense vibrations that can fracture structures during very long periods of exposure as stated in [16], this consequently leads to fatigue and loss of concentration [2].

In real-world applications, the characteristic of the acoustic environment is often varying, and so the frequency, amplitude, and phase of the noise signal are also nonstationary (timevarying), thus, an ANC system must be adaptive in order to deal with these varying characteristics [4], [17]. The most commonly used adaptive filters are realized using a Finite Impulse Response (FIR) filter with the Least Mean Square (LMS) algorithm [14]. Narula et al. proposed an effective Adaptive filtering technique in [18], which utilize a single channel negative feedback control using LMS adaptive filters to remove unwanted noise in a real-time environment. A higher convergence rate than the proposed algorithm in [19] is depicted, where Sukhpreet et al. analyse the capability of the algorithm in mitigating different Signal to Noise Ratio (SNR). Both simulation and experimental results obtained in [19] reveals effective filtering of the noise in higher SNR signal with $15 \mathrm{~dB}$ reduction, however, it can't mask the noise in low SNR signal. Moreover, another work in [14] implements LMS adaptive filter algorithm to analyse the range of frequency noise for which it can mask in the corrupted signal. Result obtained has proven that this proposed algorithm can effectively mitigate lower noise frequencies i.e., $(0 \mathrm{~Hz}$ to $5000 \mathrm{~Hz})$ in high SNR signal.

To solve the issue of mitigating low-frequency noise in low SNR signal, Sen $\mathrm{M}$ et al in [4] modified the Adaptive filter algorithm in [5], [16]. This algorithm is designed to form a standing wave for the noise signal, whereby an estimate of the original noise with $180^{\circ}$ phase shift is generated and propagate in the same direction with the original noise to cancel out [20]. The ANC performance in this technique is mainly dependent on the accuracy of the amplitude and phase of the anti-noise generated by a signal processing algorithm [21]. However, the implementation of ANC is complicated although it has high reliability in cancelling noise. Initial ANC project with simple inverter fails, due to its low rate of sampling capability and unable to propagate sound wave in all direction [22]. This leads to use LMS adaptive filter with an external update algorithm [20]. Most recent articles utilise Normalize form of the LMS to achieve more stable and faster convergence condition than the simple LMS as in [15], which use Normalizes LMS adaptive filter with fixed step size to mitigate the effect of transformer noise of less than $1000 \mathrm{~Hz}$. On contrary, the Filtered LMS (FXLMS) algorithm requires a filtered version of the reference signal as input in which the filter is having the same impulse response as the cancellation path. FXLMS had been used on cancelling the periodic noise generated by laptop fan after identification [14]. Recently, Yang et al. [23] reduced 8dB noise level in the cabin of a high-speed elevator by implementing modified FXLMS algorithm. Nevertheless, the FXLMS algorithm has complex computation due to the consideration of the secondary path filter. ANC had been also used in the microcontroller in [24] which reduce an extreme low-frequency noise of 50-60 Hz, obtained results in both cases show a 20$25 \mathrm{~dB}$ noise mitigation. The steady-state error of the Normalize LMS algorithm with a fixed step-size is very large for a nonstationary input, thus, Hamidia et al. [25] improved the algorithm performance using a variable step-size, results obtained present decrease in this steady-state error by $3 \mathrm{~dB}$ from that obtained using fixed step size.

In this paper, a comparative analysis of ANC headphones which utilize different models used in the state of art including Adaptive filtering and Standing wave technique is performed. In the former one, the model structure used in [14] was adopted, whereas in the latter one model scheme used in [4] was adopted. Two different sampled frequency speech signals are used as the desired signal. Sensitivity analysis based on two broadband frequency noise of $0-1000 \mathrm{~Hz}$ and $1000-2000 \mathrm{~Hz}$ was done and also based on different SNR. The frequency range of the successfully cancelled noise by the LMS adaptive filter algorithm is determined by performing Fast Fourier Transform (FFT) on the signals. The performance of the designed methods was evaluated using convergence rate and masked noise SLP.

\section{ANC structure}

\subsection{Adaptive filtering in $\mathrm{ANC}$}

An adaptive filter is a digital filter that has self-adjusting features. It is capable of regulating its transfer function coefficients automatically to adopt the input signal through an LMS update algorithm [26]. Adaptive filters play a significant role in modern technological products in areas such as telephone echo cancellation and ANC headphones. Adaptive filters work generally for the adaptation of signal-varying environments, spectral overlap between noise corrupted signal and unknown (time-varying) noise [14]. For example, when the interference noise is a high or low-frequency noise, and its spectrum overlaps some of the frequency of the desired signal, removing the interference using traditional filter such as a high pass or low pass filter with the fixed filter coefficients will fail to preserve the desired signal spectrum.

The concept of noise filtering using an Adaptive filter is depicted in Fig. 1, and its basic operation is summarised as follows:

- Varying external unknown noise is estimated by the LMS estimator

- $\quad$ The adaptive filter adjusts its filter coefficient to filter out the noise frequencies in the corrupted signal with good preservation of the desired signal spectrum.

- Human ear receives desired speech signal spectrum with little noise residual

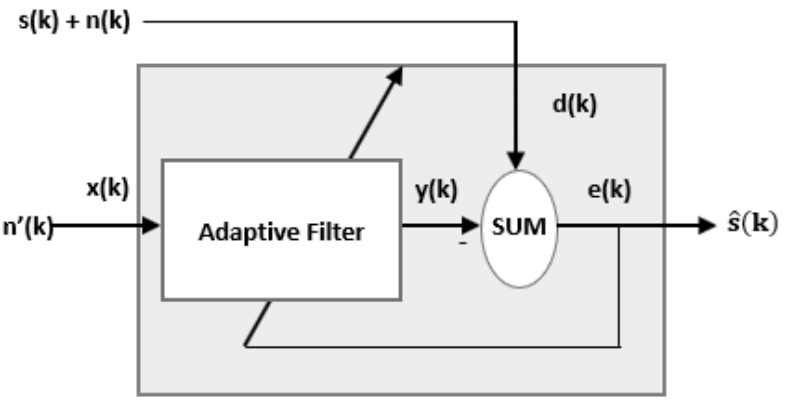

Fig. 1. ANC with adaptive filtering 


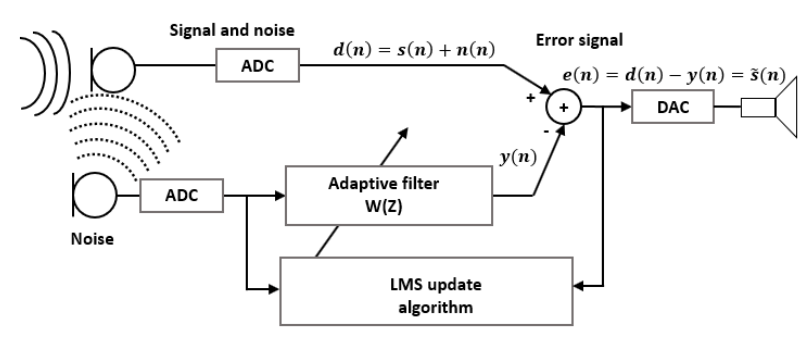

Fig. 2. ANC with adaptive filtering control scheme

Figure. 2 represent the control scheme of ANC headphones using adaptive filtering [14], it consists of two microphones. The first one sampled the desired speech $\mathrm{s}(\mathrm{n})$ to capture its components. However, due to a noisy environment, the signal is contaminated which makes the Analogue to Digital converter (ADC) channel to produces a noise-corrupted signal; that is, $d(n)=s(n)+n(n)$. The second microphone is placed at the back of the headphones' ear-cup where outside environmental noise is picked up by ADC channel to captures unknown noise $x(n)$, which is fed to the adaptive filter $W(z)$. Note that the corrupting noise $n(n)$ in the first channel is uncorrelated to the desired signal $\mathrm{s}(\mathrm{n})$, so that separation between them is possible. The noise signal $\mathrm{x}(\mathrm{n})$ from the secondary path is correlated to the corrupting noise $\mathrm{n}(\mathrm{n})$ in the primary path, since both come from the same noise source. Similarly, the noise signal $\mathrm{x}(\mathrm{n})$ is not correlated to the desired speech signal $\mathrm{s}(\mathrm{n})$. When the noise estimate $y(n)$ equals to the noise $n(n)$ in the corrupted signal, that is, $\mathrm{y}(\mathrm{n}) \approx \mathrm{n}(\mathrm{n})$, the error signal $\mathrm{e}(\mathrm{n})=\mathrm{d}(\mathrm{n})$ $\mathrm{y}(\mathrm{n})=\tilde{s}(\mathrm{n})$ will approximate the desired speech signal $\mathrm{s}(\mathrm{n})$. Hence, the noise is removed in the desired signal. Note that in this application, the error signal actually converges to the desired speech signal, rather than converging to zero.

The filter coefficient $\mathrm{W}(\mathrm{z})$ is adjusted based on the LMS algorithm in Eq.1 [18]:

$$
\mathrm{w}(\mathrm{n}+1)=\mathrm{w}(\mathrm{n})+\mu \mathrm{e}(\mathrm{n}) \mathrm{x}(\mathrm{n})
$$

where $w(n)$ is the coefficient response used currently, while $w$ $(n+1)$ is the coefficient response obtained from the LMS algorithm and will be used for the next coming input sample. The discrete form of the estimated noise and the coefficient response are presented in Eq. 2 and Eq. 3 respectively where $\mathrm{N}$ is the number of samples:

$$
\begin{aligned}
& x(n)=[x(n) x(n-1) \cdots x(n-N+1)] \\
& w^{T}(n)=\left[w_{0}(n) w_{1}(n) \cdots w_{n-1}(n)\right]
\end{aligned}
$$

The control output response is described as in Eq.4:

$$
y(n)=w^{T}(n) x(n)
$$

Assuming that $\mathrm{W}(\mathrm{z})$ has sufficient order, after the convergence of the adaptive filter, the residual error is approximately the desired speech signal $(\mathrm{e}(\mathrm{n})=\tilde{s}(\mathrm{n}))$ [14]. That is control output response is close to the noise free signal.

\subsection{Standing Wave Pattern in ANC}

In ANC using the standing wave technique, its basic operation is that there is a microphone, which captures the external noise and then performs some computation to generate an identical noise signal in opposite phase, this allows it to cancel the original noise as seen in [4]. In reality, this doesn't mitigate the noise to zero but reduces it by a considerable amount close to zero. The use of adaptive filters is one of the effective ways in standing wave pattern because of its capability to respond to changes in the noise parameters. This behaviour is possible because the adaptive filter coefficients vary over time and update automatically by an external LMS update algorithm [15]

The LMS update algorithm is an approximation of the steepest descent algorithm which uses an instantaneous estimate of the gradient vector of the error function. The estimate of the gradient is based on sample values of the tap-input vector and an error signal [6]. The initial idea is to optimize the FIR filter weights "on-line" based on the adaptive filter LMS algorithm to develop an adaptive filter. Here, an adaptive filter is needed that is capable of estimating noise signal, altering its impulse response as required for the ANC headphone application. The concept of standing wave pattern using Adaptive filter is depicted in Fig. 3, and its basic operation is summarised as follows:

- Varying external unknown noise is estimated by the LMS estimator

- Invert the estimated noise with a phase shift of at least $90 \%$. Then propagate it in the opposite direction (addit) to the original noise signal to form a standing wave.

- The adaptive filter adjusts its filter coefficient to minimize the resulting error to zero by optimizing the amplitude, phase angle of the estimated noise.

- Human ear receives desired speech signal spectrum with little noise residual

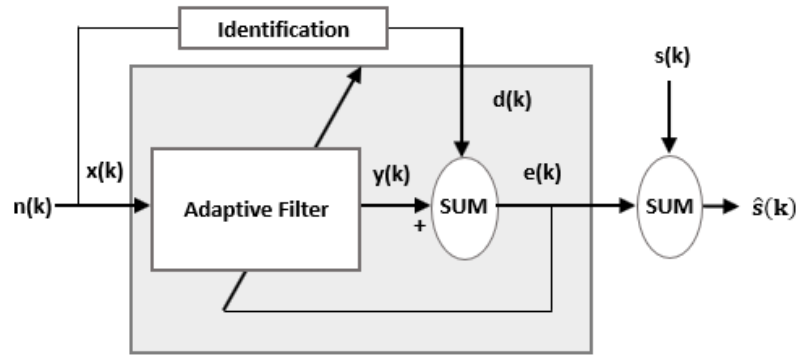

Fig. 3. ANC with Standing wave pattern

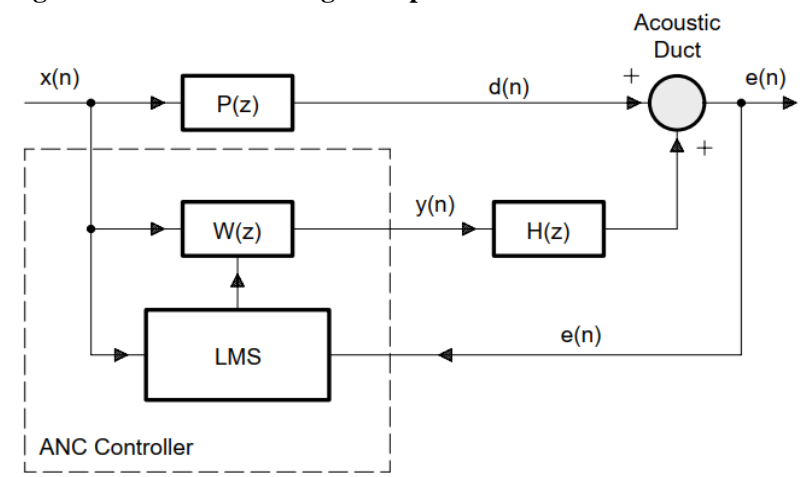

Fig. 4. ANC with Standing wave pattern control scheme

Figure. 4 represent the control scheme of ANC headphones using standing wave pattern [21], it also consists of two microphones that are placed at the back of the headphones' earcup to picked up outside noise and noise residual. An ADC sampled the noise $\mathrm{x}(\mathrm{n})$ from the reference microphone and then is fed to the adaptive filter $\mathrm{W}(\mathrm{z})$. The adaptive filter $\mathrm{W}(\mathrm{z})$ estimate the response of the unknown primary acoustic path $\mathrm{P}(\mathrm{z})$ between the reference microphone and the error microphone. And also estimate the response of the unknown secondary acoustic path $\mathrm{H}(\mathrm{z})$ between the cancelling speaker and the error microphone. The noise signal $\mathrm{x}(\mathrm{n})$ from the secondary path is correlated to the original noise $\mathrm{d}(\mathrm{n})$ in the primary path since both come from the same noise source. When the estimated noise $y(n)$ has the same amplitude and $180^{\circ}$ out of phase with 
desired noise $\mathrm{d}(\mathrm{n})$, that is, $\mathrm{y}(\mathrm{n}) \approx-\mathrm{d}(\mathrm{n})$, the error signal $\mathrm{e}(\mathrm{n})=$ $\mathrm{d}(\mathrm{n})-\mathrm{y}(\mathrm{n})=0$. Hence, the original noise is cancelled with the estimated noise using the concept of destructive interference. Note that in this application, the error signal converges to zero depending on the accuracy of the amplitude and phase of the anti-noise generated.

Using a digital frequency-domain representation of the problem in Fig. 5, the ANC system uses an adaptive filter $\mathrm{W}(\mathrm{z})$ to estimate the response of an unknown primary acoustic path $\mathrm{P}(\mathrm{z})$ and the unknown secondary acoustic path $\mathrm{H}(\mathrm{z})$ [15]. The $\mathrm{z}$ transform of e(n) can be expressed as in Eq.5:

$$
\mathrm{E}(\mathrm{z})=\mathrm{X}(\mathrm{z}) \mathrm{P}(\mathrm{z})+\mathrm{X}(\mathrm{z}) \mathrm{W}(\mathrm{z}) \mathrm{H}(\mathrm{z})
$$

Assuming that $\mathrm{W}(\mathrm{z})$ has sufficient order, after the convergence of the adaptive filter, the residual error is zero (that is, $e(n)=0$ ). This result requires $\mathrm{W}(\mathrm{z})$ to be as in Eq.6.

$$
\mathrm{W}(\mathrm{z})=-\frac{\mathrm{P}(\mathrm{z})}{\mathrm{H}(\mathrm{z})}
$$

The filter coefficient $\mathrm{W}(\mathrm{z})$ is adjusted based on the LMS algorithm in Eq.7:

$$
\mathrm{w}(\mathrm{n}+1)=\mathrm{w}(\mathrm{n})-\mu \mathrm{e}(\mathrm{n}) \mathrm{x}(\mathrm{n}) \mathrm{h}(\mathrm{n})
$$

where $w(n)$ is the coefficient used currently, while $w(n+1)$ is the coefficient obtained from the LMS algorithm and will be used for the next coming input sample. With discrete form of the estimated noise and the filter coefficient response in Eq. 8 and Eq.9 respectively, where $\mathrm{N}$ represent the total number of samples.

$$
\begin{aligned}
& x(n)=[x(n) x(n-1) \cdots x(n-N+1)] \\
& w^{T}(n)=\left[w_{0}(n) w_{1}(n) \cdots w_{n-1}(n)\right]
\end{aligned}
$$

The control output response is depicted in Eq.10:

$$
y(n)=\sum_{i=0}^{N-1} w_{i}(n) x(n-i)
$$

At any frequency $\mathrm{w}$, such that $\mathrm{H}(w)=0$, the control system become Unstable. And also, at any frequency w, such that $\mathrm{P}(w)=0$, the control system becomes Ineffective. To avoid risking the system to an unstable state, Kuo et al. in [4] modified the control scheme in [16] as shown in Fig. 6

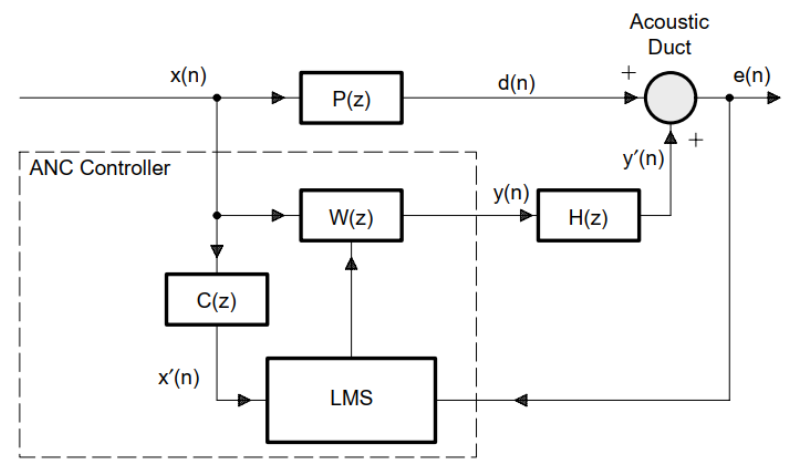

Fig. 5. Modified ANC headphone control scheme

At last, from Fig. 6, C(z) will compensate effect of having H(z) $=0$. The model modified the LMS update algorithm while leaving all order response the same, as presented in Eq.11:

$$
w(n+1)=w(n)-\mu e(n) x^{\prime}(n) h(n)
$$

The discrete form of the filter response $\mathrm{C}(\mathrm{z})$ and its corresponding output are presented in Eq. 12 and Eq.13 respectively.

$$
\begin{aligned}
& \mathrm{c}^{\mathrm{T}}=\left[\mathrm{c}_{0}(\mathrm{n}) \mathrm{c}_{1}(\mathrm{n}) \cdots \mathrm{c}_{\mathrm{n}-1}(\mathrm{n})\right] \\
& \mathrm{x}^{\prime}(\mathrm{n})=\sum_{i=0}^{N-1} c_{i}(n) x(n-i)
\end{aligned}
$$

\section{Methodology}

Reduction of noise has become inevitable, various approaches are discussed in the literature, ranging from the older to recent ones. Having the discussed ANC structure, the recent and efficient ANC techniques used in [4], [14] were adopted. And some designed specifications are added. Which include the following:

i. Optimization of the step size and the filter length of the LMS adaptive filter while maintaining system stability and faster rate of convergence: this is to improve the noise cancellation in the transient state.

ii. Addition of sampling rate improves the estimation power of the filter, by reducing the delay caused in the anti-noise phase.

Firstly, two audio files named 'Audio A' and 'Audio B' of Waveform Audio (wav) Format is used as the desired speech signal and Gaussian random noise $($ mean $=0$, variance $=[0.01$ 1] is used as the outside noise to be masked. The ANC simulation models are developed in MATLAB/SIMULINK software environment as structured in Fig 2 and Fig 4. For the purpose of sensitivity analysis, the following are used:

- $\quad$ Broadband (random) noise signal of (0 -1000) Hz and (1000- 2000) Hz

- $\quad$ Signal to Noise ratio (SNR) of 40,30, and 20dB

- Audio signal sampling rate of $8000 \mathrm{~Hz}$ and $48000 \mathrm{~Hz}$ for ' $A$ ' and ' $B$ ' respectively.

The power of the audio signals $P_{S}$ is computed using the relation in Eq.14 whereas the power of Gaussian noise $P_{\text {noise }}$ is approximated by the expression in Eq.15 [27], with $x$ as the audio signal, $\mathrm{N}$ total number of sample and $\sigma$ standard deviation of the noise with zero mean:

$$
\begin{aligned}
& \mathrm{P}_{\mathrm{S}}=\frac{\sum_{\mathrm{i=1}=1}^{\mathrm{N}} \operatorname{norm}\left(\mathrm{x}_{\mathrm{i}}^{2}\right)}{\mathrm{N}} \\
& \mathrm{P}_{\text {noise }}=\sigma^{2}
\end{aligned}
$$

The SNR of an audio signal is express in term of signal power and noise power as in Eq.16 [28]:

$$
\mathrm{SNR}=20 \log \left(\frac{\mathrm{P}_{\mathrm{S}}}{\mathrm{P}_{\text {noise }}}\right)
$$

The Sound Pressure Level (SPL) is one of the main factors to be considered in noise reduction system [29], it is express in Eq.17 in term of root mean square value of the signal $P_{r m s}$ and threshold of human hearing $P_{\text {ref }}$.

$$
\mathrm{SLP}=20 \log \left(\frac{\mathrm{P}_{\mathrm{rms}}}{\mathrm{P}_{\mathrm{ref}}}\right)
$$

In DSP, Fast Fourier Transform (FFT) is one of the most useful tools in signal processing, as such it is employed to see the effect of the noise frequency removal. The FFT of a signal says $x$ [30], is given in Eq.18: 


$$
\mathrm{X}_{\mathrm{k}}=\sum_{\mathrm{n}=0}^{\mathrm{N}-1} \mathrm{X}_{\mathrm{n}} \mathrm{e}^{-\mathrm{i} 2 \pi \mathrm{kn} / \mathrm{N}}, \quad \mathrm{k}=0,1, \ldots, \mathrm{N}-1
$$

\subsection{ANC with Adaptive filter problem formulation}

In this control scheme, the Adaptive filter is configured with Two input signals and One output signal as described in Fig 1. Input $\mathrm{y} 1$ is the desired signal which is taken as the noise corrupted speech signal (combination of speech signal and Gaussian random noise) and Input y2 is the Input signal which is taken as the noise signal. Another audio signal which is the speech signal (noise-free speech signal), s(n) is used as a reference signal for comparison with the filtered output of the algorithm, e(n). Thus, the human ear receives the filtered output signal, e(n). The LMS algorithm is initiated by setting the column weight vector $w(n)$ and the filtered output e(n) as a zerocolumn vector.

The initial value for the step size $(\mu=0.04)$ is calculated from the stability condition criteria in Eq.19, in term of the filter length $\mathrm{N}$ and power of the white noise $P_{\text {noise }}$ :

$$
0 \leq \mu \leq \frac{1}{\mathrm{NP}_{\text {noise }}}
$$

Upon sending Input $y 1$ and Input $y 2$ to the LMS adaptive filter block. Input $y 2$ is multiplied by the column weight vector $\mathrm{w}(\mathrm{n})$ to generate a weighted-noise signal $y 2$ '. The intermediate output $y 2^{\prime}$ is then subtracted from the Input $y 1$ to obtain the error e (n). After each interaction, the column weight vector would adaptively update. The filtering process would be continuing until it reaches the prescribed filter length $\mathrm{N}$, which is the same as the number of input sample. The absolute difference between the filtered output $\mathrm{e}(\mathrm{k})$ of the LMS adaptive filter algorithm and the reference input (noise-free speech signal) is calculated as the residual.

For performance evaluation of the designed ANC, FFT in Eq. 18 is applied on both the filtered and noisy signal to obtain their correspondence frequency response. Also, the time response of the noise-free speech signal, filtered output and the residual between them is analysed. Moreover, the SLP of the noisy and filtered signal is computed using Eq.17, to find the reduced SLP.

\subsection{ANC with standing wave problem formulation}

To form an effective control of standing wave generation in ANC, the Adaptive filter is configured as an estimator, with Two input signal and One output signal as described in Fig 4. Input $\mathrm{y} 1$ is the desired signal which is taken as the identified noise between the reference noise microphone and the error microphone and Input y 2 is the Input signal which is taken as the reference noise signal. The desired signal is compared with the Input signal to generate the error signal, e(n) using the concept of destructive interference. The LMS algorithm will take in this error signal to update the Adaptive filter w(n) coefficient, non-zero error is obtained if there is magnitude and phase difference between the signals.

Human ears receive an audio signal which is the speech signal (noise-free speech signal), s(n) and small noise residual, e(n). Here, also the algorithm is initiated by setting the column weight vector $w(n)$, primary path $p(n)$, secondary path $s(n)$, and antisecondary path $\mathrm{c}(\mathrm{n})$ as a zero-column vector, with the same initial value for the step size $(\mu=0.002)$ used in the first case. Upon sending Input $y 1$ and Input $y 2$ to the LMS adaptive filter block. The adaptive filter performs off-line system identification to find the unknown paths $\mathrm{p}(\mathrm{n}), \mathrm{h}(\mathrm{n})$, and $\mathrm{c}(\mathrm{n})$ response. Input $y 2$ is multiplied by the column weight vector $w(n)$ to generate a weighted-noise signal $y 2$ '. Which is then scale-up with $\mathrm{h}(\mathrm{n})$ to obtained estimated noise of the same magnitude and $180^{\circ}$ out of phase with the desired signal. After the destructive interference between the two signals, the residual noise is computed, e(n), and used to adaptively updates the LMS algorithm. This process would be continuing until it reaches the prescribed filter length $\mathrm{N}$, which is the same as the number of input sample.

The frequency range of the successfully cancelled noise by the LMS adaptive filter algorithm is determined by performing FFT on the required signals. Also, the time response of the noise-free speech signal, residual noise, and human received signal were analysed to evaluate the performance of the designed ANC. Besides, the SLP of the noisy and filtered signal is computed using Eq.17, to find the reduced SLP.

\section{Results and Discussion}

In this section, the results obtained from the ANC simulated techniques are discussed and evaluated based on the noise reduction in speech signals, and frequency range of the successfully cancelled noise.

The step size and the filter length are intrinsically related to the performance of adaptive filtering, with the length of the filter specifically responds to the accuracy of LMS adaptive filter modelling and step size response to the stability of the filter model. Due to the fact that this filter length affects the rate of convergence through the computational time. The trade-off between the filter length and step size is that an increase in the filter length increased the iteration and the convergence rate is decreased. Firstly, the value of step size is fixed at 0.04 and then the filter length $\mathrm{L}$ effect is studied. Chosen values for $\mathrm{L}$ are; 20 , $25,30,40$ and 50. The results obtained shows that the convergence of error signal increases until the $\mathrm{L}$ is 40 , at this value the model starts diverging and noise reduction reduced. Furthermore, the filter length $L$ is kept fixed at 40 and step sizes are varied within the stability condition mention in section 3.1, as $0.002,0.01,0.02$ and 0.05 . Result shows that the filter convergence rate decrease at 0.002 and the convergence rate keep rising until the system reaches 0.04 where divergence starts. But to my surprise, the best convergence is at a step size of 0.031 . Hence, it can be concluded that the optimal values of the Filter length and step size are 40 and 0.031 respectively.

To investigate the sensitivity of frequency in both the two proposed ANC techniques, reduction of monotonic noise signal of 250,500 , and $1000 \mathrm{~Hz}$ are depicted as shown in Fig. 6 . Obtained shows that standing wave pattern has better noise reduction at a frequency lower than $650 \mathrm{~Hz}$ than the adaptive filtering technique. Whereas the adaptive filtering mitigates the noise at high frequency than standing wave as shown in Fig. 6.

This is not surprising as it is well known that standing wave pattern accuracy decrease with an increase in the frequency of the signal. 

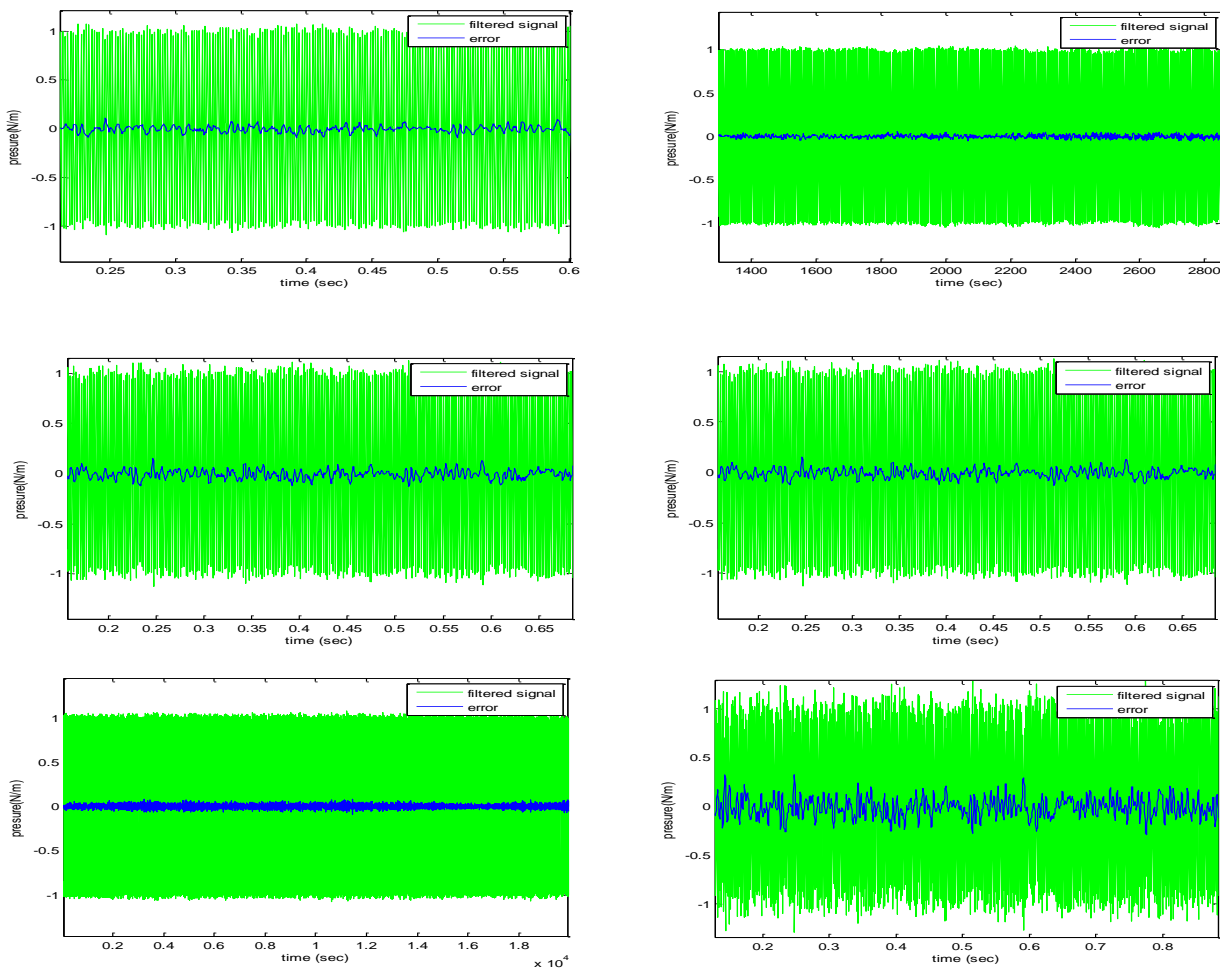

Fig. 6. ANC Sensitivity to monotonic frequencies

The audio signals sampled at $8 \mathrm{kHz}$ and $48 \mathrm{kHz}$ for $\mathrm{A}$ and $\mathrm{B}$ respectively were used to evaluate the proposed techniques, and the recorded time is $10 \mathrm{~s}$. The FFT of these audio signals is shown in Fig. 7. Most audio signals such as speech signal, music signal are typical non-stationary signals, to achieve a good performance, the speech signals must be segmented. The audio signal is segmented every $40 \mathrm{~ms}$ and a frame includes 32 sampled data. In the simulation, broadband Gaussian noise of different variance $\sigma^{2}$ is added to the audio signals, to have $40 \mathrm{~dB}$, $30 \mathrm{~dB}$ and $20 \mathrm{~dB}$ SNR.

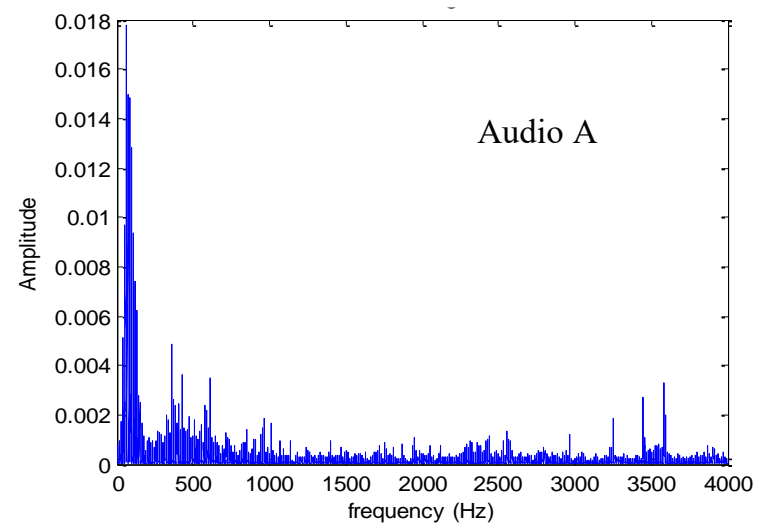

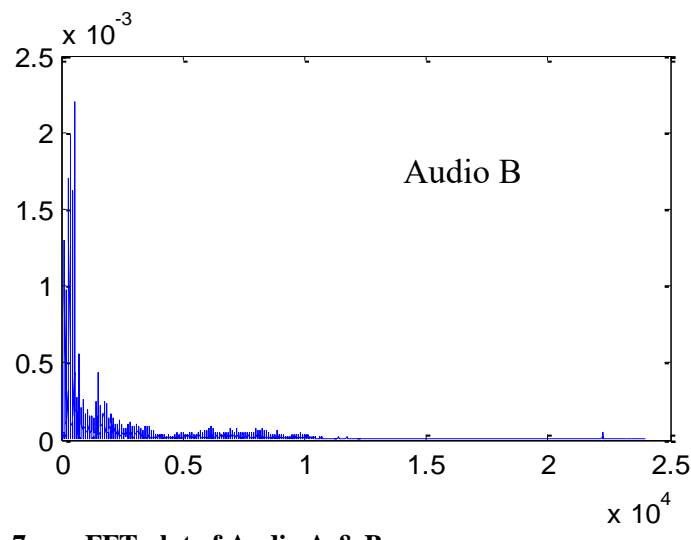

Fig. 7. FFT plot of Audio A \& B

\footnotetext{
* Corresponding author. Tel.: +1234567890

Fax: +9876543210; E-mail: Author.Name@iasks.org

(C) 2016 International Association for Sharing Knowledge and Sustainability DOI: 10.5383/ijtee.17.02.001
} 
(a)
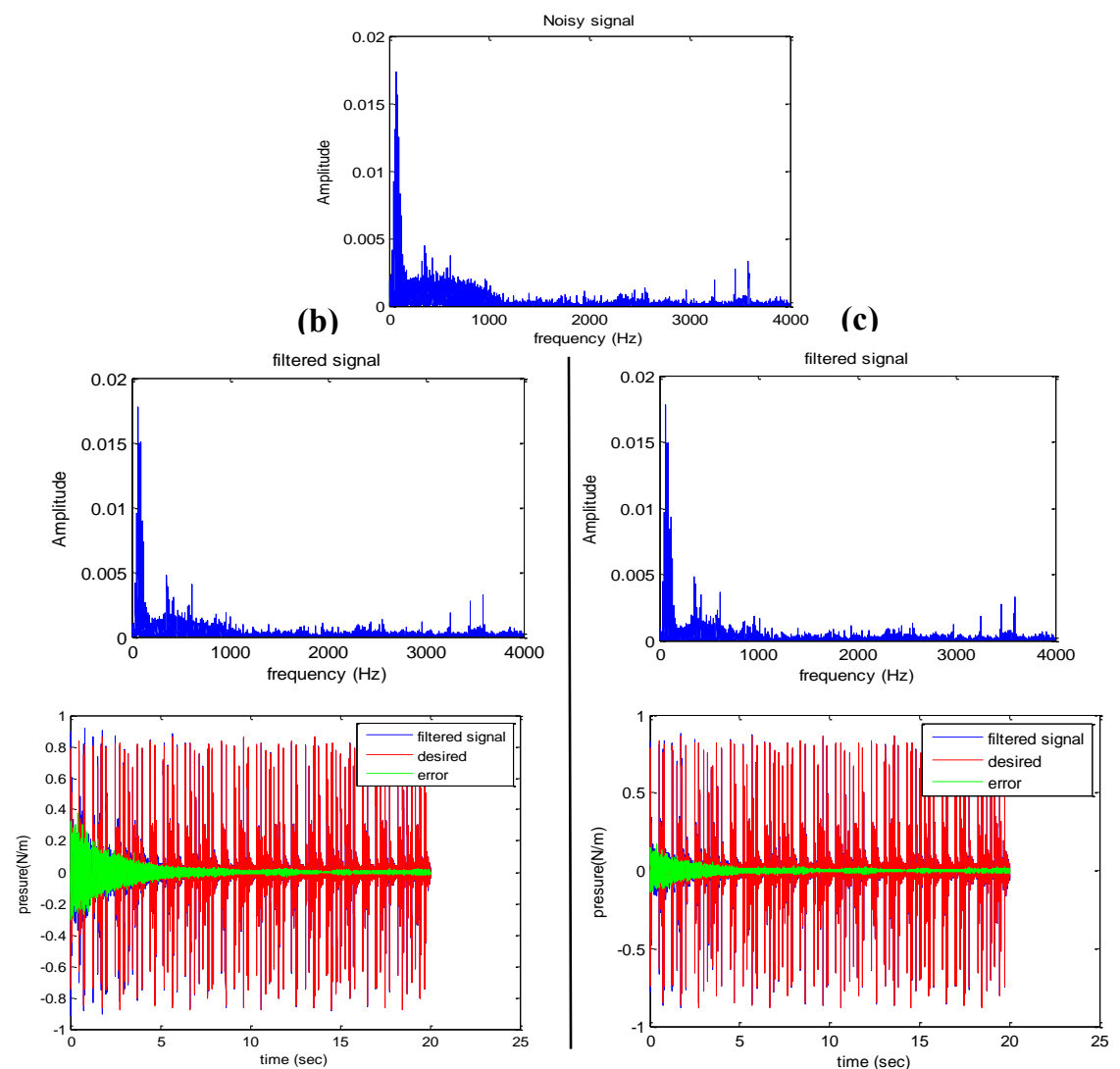

Fig. 8. SNR- $40 \mathrm{~dB} /$ Audio A/ Noise (1k - 2kHz) (a) Noisy signal FFT, (b) Adaptive filtering performance and (c) standing wave performance.

(a)
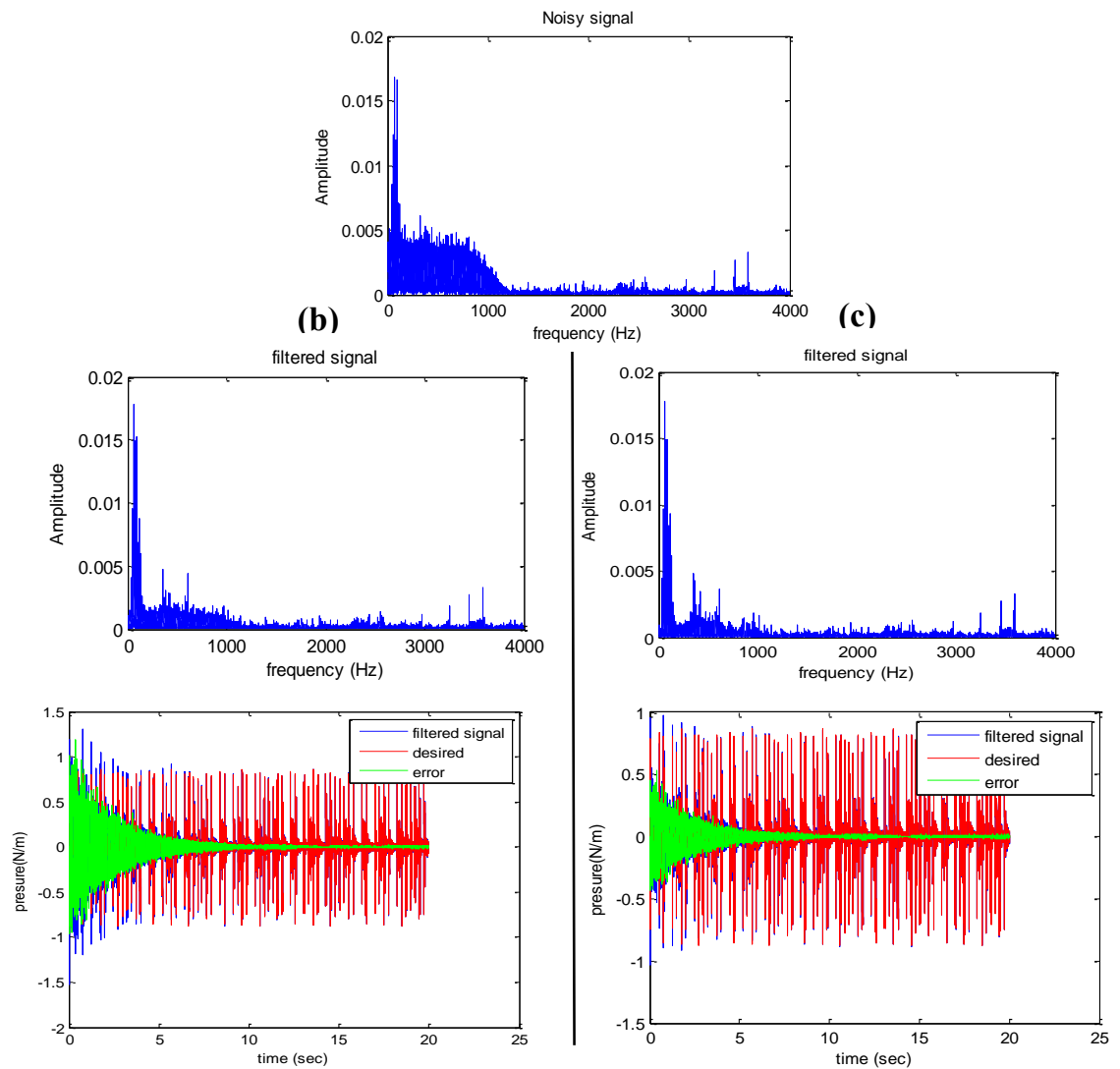
Fig. 9. SNR- $40 \mathrm{~dB} /$ Audio A/ Noise (1k - 2kHz) (a) Noisy signal FFT, (b) Adaptive filtering performance(c) standing wave performance.

(a)
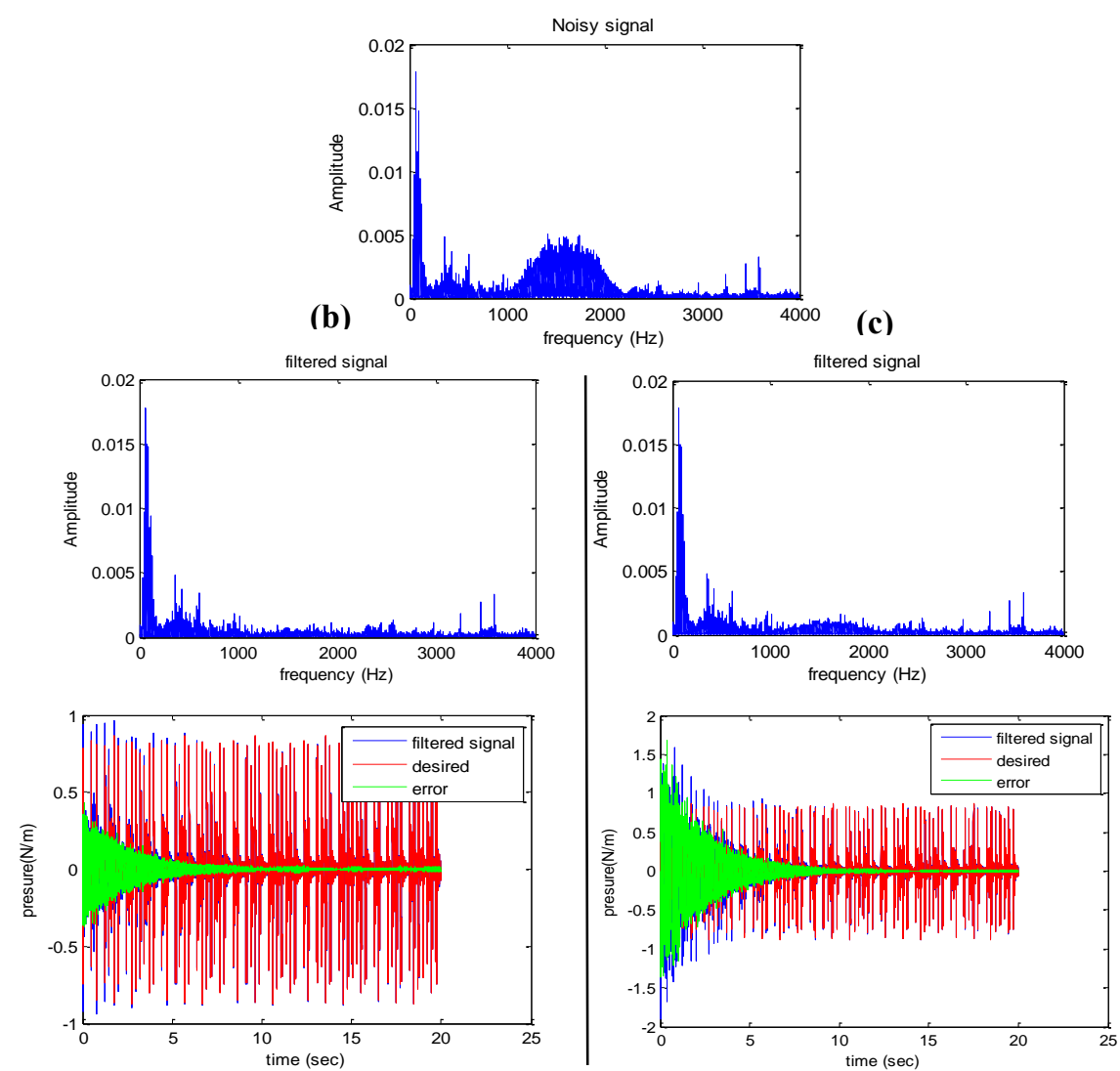

Fig. 10. SNR- $40 \mathrm{~dB} /$ Audio A/ Noise (1k - 2kHz) (a) Noisy signal FFT, (b) Adaptive filtering performance and (c) standing wave performance.

(a)

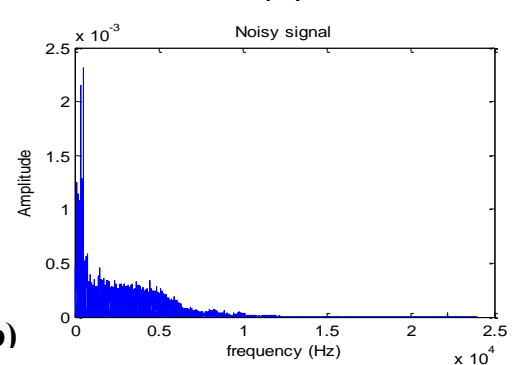

(c)
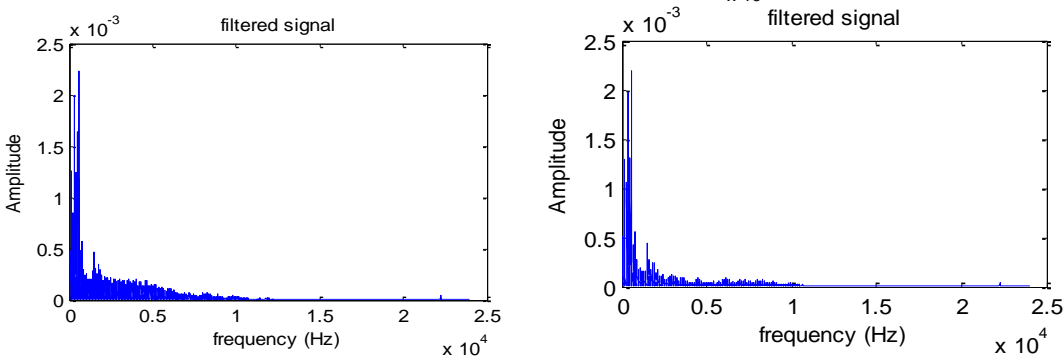

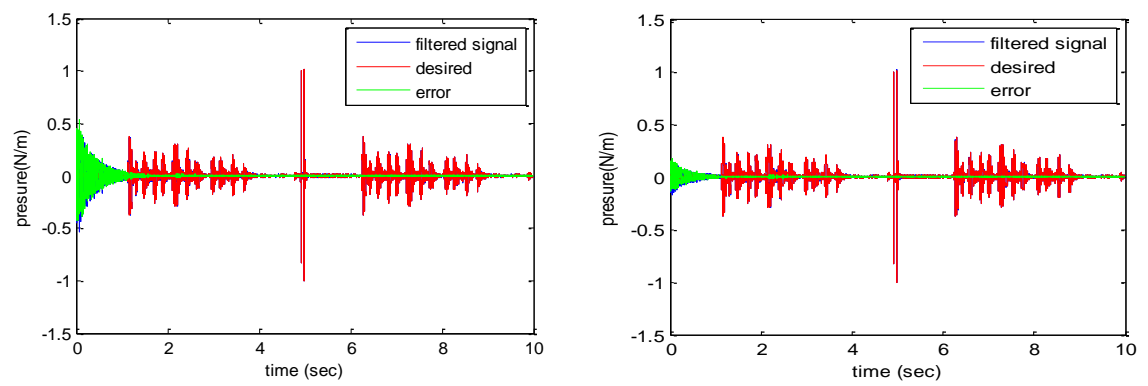

Fig. 11. SNR- $40 \mathrm{~dB} /$ Audio B/ Noise (1k-2kHz) (a) Noisy signal FFT, (b) Adaptive filtering performance and (c) standing wave performance.

From the results obtained in Fig. 8, it can be seen that for a noisy signal of $40 \mathrm{~dB}$ SNR (audio A corrupted with a broadband noise frequency of $0-1000 \mathrm{~Hz}$ ), the standing wave pattern ANC has better convergence rate of $3.2 \mathrm{sec}$ than that of adaptive filtering ANC $7.2 \mathrm{sec}$, with a measured noise reduction of $52.78 \mathrm{~dB}$ and $41.66 \mathrm{~dB}$ respectively. So also, from Fig. 9 with a noisy signal of $30 \mathrm{~dB}$ SNR corrupted with the same frequency range, the standing wave pattern has better convergence rate of $4.8 \mathrm{sec}$ than that of adaptive filtering $10.2 \mathrm{sec}$ having a measured noise reduction of $49.78 \mathrm{~dB}$ and $22.36 \mathrm{~dB}$ respectively. To emphasis the obtained result in Fig. 8, another $40 \mathrm{~dB}$ noisy signal is generated from (audio B corrupted with a broadband noise frequency of $0-1000 \mathrm{~Hz}$ ) as depicted in Fig. 11, the standing wave ANC has better convergence rate and noise reduction than adaptive filtering ANC. Thus, we can say that standing wave technique has better performance for a low SNR signal than adaptive filtering, but of course for a broadband noise frequency less than $800 \mathrm{~Hz}$

Changing the broadband frequency range of the Gaussian white noise to $1000-2000 \mathrm{~Hz}$. It can be seen that from Fig. 10 noisy signal of $40 \mathrm{~dB}$ generated from audio A. Adaptive filtering ANC has better convergence rate of $7.8 \mathrm{sec}$ than that of standing wave pattern ANC $11.6 \mathrm{sec}$, with a measured noise reduction of 40.12 $\mathrm{dB}$ and $19.11 \mathrm{~dB}$ respectively. When compared with the same Noisy signal in Fig. 8 but different frequency band, it can see that their performance using the Adaptive filtering method is very close, while that of the Standing wave greatly differ. We have done the sensitivity analysis based on different SNR and different frequency band as presented in Table 1 .

Table 1. Sensitivity Analysis based on SNR and frequency band

\begin{tabular}{|c|c|c|c|c|}
\hline Methods & $\begin{array}{l}\text { SNR } \\
\text { (dB) }\end{array}$ & $\begin{array}{l}\text { Frequency } \\
\text { band }(\mathrm{Hz})\end{array}$ & $\begin{array}{l}\text { Convergence } \\
\text { rate (sec) }\end{array}$ & $\begin{array}{l}\text { Masked } \\
\text { SLP (dB) }\end{array}$ \\
\hline \multirow{3}{*}{$\begin{array}{l}\text { Adaptive } \\
\text { filtering }\end{array}$} & 40 & & 7.2 & 41.66 \\
\hline & 30 & & 10.5 & 22.36 \\
\hline & & $0-1000$ & & \\
\hline \multirow{2}{*}{$\begin{array}{l}\text { Standing } \\
\text { wave } \\
\text { pattern }\end{array}$} & 40 & & 3.2 & 52.78 \\
\hline & 30 & & 5.0 & 49.78 \\
\hline
\end{tabular}

\begin{tabular}{|c|c|c|c|c|}
\hline \multirow{2}{*}{$\begin{array}{l}\text { Adaptive } \\
\text { filtering }\end{array}$} & 40 & & 7.8 & 40.12 \\
\hline & 30 & & 11.6 & 19.11 \\
\hline \multirow{2}{*}{$\begin{array}{l}\text { Standing } \\
\text { wave } \\
\text { pattern }\end{array}$} & 40 & $1000-2000$ & 11.2 & 22.12 \\
\hline & 30 & & 14.8 & 10.92 \\
\hline
\end{tabular}

From the run sensitivity analysis tabulated in Table. 1, it can be concluded that standing wave pattern has the better performance at mitigating noise frequency below $800 \mathrm{~Hz}$, with low SNR than Adaptive filtering. However, cannot be used to masked highfrequency noise (i.e., reduction less than $11 \mathrm{~dB}$ ). Despite the fact, Adaptive filtering has low performance for a low SNR at all frequencies but it can still mask some high frequencies up to $22 \mathrm{~dB}$.

\section{Conclusion}

In this paper, real-time simulation of Active Noise Cancellation (ANC) algorithm was designed using the start of art techniques discussed including adaptive filtering and standing wave pattern. The control schemes were structure based on single-channel broad-band feedforward and feedback control, utilizing Adaptive filter with Least Means Square (LMS) estimation, in SIMULINK framework. Speech signals were used as the desired audio signal and Gaussian white noise of zero mean uniform distribution as the outside noise. To generate different SNR signals with a fixed speech signal, the Gaussian noise variance $\sigma^{2}$ was varied from $(0.01-1)$. Gradient descents method was employed to improve the adaptation capability and convergence rate of the LMS adaptive filter. This yields an optimal value of the filter length $L$ and step size $\mu, 40$ and 0.031 respectively. From the run sensitivity analysis on both the designed models, it can be concluded that ANC headphone using standing wave pattern has the better performance at mitigating noise frequency below $800 \mathrm{~Hz}$, with low SNR than those using Adaptive filtering. However, ANC headphone using standing wave pattern cannot be used to masked high-frequency noise (i.e., reduction less than $11 \mathrm{~dB})$. On the other hand, ANC headphone using Adaptive filtering has low performance for a low SNR at all frequencies but it can still mask some high frequencies up to $22 \mathrm{~dB}$. 


\section{References}

[1] S. D. Gordon and G. W. Uetz, "Environmental interference: Impact of acoustic noise on seismic communication and mating success," Behav. Ecol., vol. 23, no. 4, pp. 707-714, 2012, doi: 10.1093/beheco/ars016.

[2] J. W. Gu, C. F. Halpin, E. C. Nam, R. A. Levine, and J. R. Melcher, "Tinnitus, diminished sound-level tolerance, and elevated auditory activity in humans with clinically normal hearing sensitivity," J. Neurophysiol., vol. 104, no. 6, pp. 3361-3370, 2010, doi: 10.1152/jn.00226.2010.

[3] A. Quadri, "A Review of Noise Cancellation Techniques for Cognitive Radio," arXiv, 2018.

[4] S. M. Kuo, S. Mitra, and W. S. Gan, "Active noise control system for headphone applications," IEEE Trans. Control Syst. Technol., vol. 14, no. 2, pp. 331-335, 2006, doi: 10.1109/TCST.2005.863667.

[5] S. M. Kuo and D. R. Morgan, "Active noise control: a tutorial review," Proc. IEEE, vol. 87, no. 6, pp. 943-973, 1999, doi: 10.1109/5.763310.

[6] A. Swain, "Active Noise Control: Basic Understanding Active Noise Control," Res. Proj. Inst. Miner. materails Technol., no. July, pp. 0-18, 2014.

[7] Y. Kajikawa, W. S. Gan, and S. M. Kuo, "Recent advances on active noise control: Open issues and innovative applications," J. Institutional Econ., vol. 1, no. 2, 2012, doi: 10.1017/ATSIP.2012.4

[8] J. Rennies and J. L. Verhey, "Temporal weighting in loudness of broadband and narrowband signals," $J$. Acoust. Soc. Am., vol. 126, no. 3, pp. 951-954, 2009, doi: 10.1121/1.3192348.

[9] J. Romeu, X. Salueña, S. Jiménez, R. Capdevila, and L. Coll, "Active noise control in ducts in presence of standing waves. Its influence on feedback effect," Appl. Acoust., vol. 62, no. 1, pp. 3-14, 2001, doi: 10.1016/S0003682X(00)00029-3.

[10] C. Wang and L. Huang, "Passive noise reduction for a contrarotating fan," J. Turbomach., vol. 137, no. 3, pp. 110, 2015, doi: 10.1115/1.4028357.

[11] S. C. Lin and C. L. Huang, "An integrated experimental and numerical study of forward-curved centrifugal fan," Exp. Therm. Fluid Sci., vol. 26, no. 5, pp. 421-434, 2002, doi: 10.1016/S0894-1777(02)00112-7.

[12] A. R. D. Curtis, P. A. Nelson, and S. J. Elliott, "Active reduction of a one-dimensional enclosed sound field: An experimental investigation of three control strategies," $J$. Acoust. Soc. Am., vol. 88, no. 5, pp. 2265-2268, 1990, doi: 10.1121/1.400353.

[13] A. Quadri, "A Review of Noise Cancellation Techniques for Cognitive Radio," J. Electr. Eng. Univ. North Dakota Gd. Forks, ND, vol. Vol 18, no. 2, pp. 168-86, 2018.

[14] J. H. Lee, L. E. Ooi, Y. H. Ko, and C. Y. Teoh, "Simulation for noise cancellation using LMS adaptive filter," IOP Conf. Ser. Mater. Sci. Eng., vol. 211, no. 1, 2017, doi: 10.1088/1757-899X/211/1/012003.

[15] M. Batool, Y. Chen, M. A. Shah, Y. Zhang, S. Wang, and M. Zhang, "Design and simulation using
MATLAB/Simulink on active noise control system for power transformer," C. 2016 - Int. Conf. Cond. Monit. Diagnosis, pp. 465-468, 2016, doi: 10.1109/CMD.2016.7757861.

[16] S. M. Kuo, I. Panahi, K. M. Chung, T. Horner, M. Nadaski, and J. Chyan, "Design of active noise control systems with the TMS320 family," Control, 1996.

[17] B. R. C. Molesworth, M. Burgess, and D. Kwon, "The use of noise cancelling headphones to improve concurrent task performance in a noisy environment," Appl. Acoust., vol. 74 , no. 1, pp. 110-115, 2013, doi: 10.1016/j.apacoust.2012.06.015

[18] V. Narula, M. Sagar, P. Joshi, P. S. Mehta, and S. Tripathi, "Real-Time Active Noise Cancellation with Simulink and Data Acquisition Toolbox," ACEEE Int. J. Control Syst. Instrum., vol. 03, no. 02, 2012.

[19] S. Singh and S. S. Sran, "Acoustic noise cancellation using block lms filter in matlab simulink," Int. J. Comput. Sci. Commun., vol. 3, no. 1, pp. 183-186, 2012.

[20] T. G. Tsuei, A. Srinivasa, and S. M. Kuo, "An adaptive feedback active noise control system," no. 1, pp. 249-254, 2002, doi: 10.1109/cca.2000.897432.

[21] E. Lopez-Caudana, P. Betancourt, E. Cruz, M. NakanoMiyatake, and H. Perez-Meana, "A hybrid noise cancelling algorithm with secondary path estimation," WSEAS Trans. Signal Process., vol. 4, no. 12, pp. 677687, 2008

[22] Q. Huang, S. Chen, M. Huang, and Z. Guo, "Adaptive Active Noise Suppression Using Multiple Model Switching Strategy," Shock Vib., vol. 2017, pp. 9-13, 2017, doi: 10.1155/2017/7289076.

[23] I. H. Yang, J. E. Jeong, U. C. Jeong, J. S. Kim, and J. E. $\mathrm{Oh}$, "Improvement of noise reduction performance for a high-speed elevator using modified active noise control," Appl. Acoust., vol. 79, pp. 58-68, 2014, doi: 10.1016/j.apacoust.2013.10.016.

[24] K. D. Wesson, R. M. Ochshorn, and B. R. Land, "Lowcost, high-fidelity, adaptive cancellation of periodic $60 \mathrm{~Hz}$ noise," J. Neurosci. Methods, vol. 185, no. 1, pp. 50-55, 2009, doi: 10.1016/j.jneumeth.2009.09.008.

[25] M. Hamidia and A. Amrouche, "Improved variable stepsize NLMS adaptive filtering algorithm for acoustic echo cancellation," Digit. Signal Process. A Rev. J., vol. 49, pp. 44-55, 2016, doi: 10.1016/j.dsp.2015.10.015.

[26] Y. Liu, Y. Liu, and M. Xiao, “A Noise Reduction Method Based on LMS Adaptive Filter of Audio Signals 3rd International Conference on Multimedia TechnologyÿÿICMT 2013),” pp. 1001-1008, 2013.

[27] J. Huillery et al., "Gaussian Noise Time-Varying Power Spectrum Estimation with Minimal Statistics To cite this version: HAL Id : hal-01062165 Gaussian Noise TimeVarying Power Spectrum Estimation with Minimal Statistics," 2014.

[28] X. Liu, J. Jia, and L. Cai, "SNR estimation for clipped audio based on amplitude distribution," Proc. - Int. Conf. Nat. Comput., pp. 1434-1438, 2013, doi: 10.1109/ICNC.2013.6818205.

[29] Y. Hao, M. C. R. Charan, G. S. Bhat, and I. M. S. Panahi, 
"Robust real-time sound pressure level stabilizer for multichannel hearing aids compression for dynamically changing acoustic environment," Conf. Rec. 51 st Asilomar Conf. Signals, Syst. Comput. ACSSC 2017, vol. 2017October, pp. 1952-1955, 2018, doi:

\subsection{9/ACSSC.2017.8335706}

[30] U. Oberst, "The fast Fourier transform," SIAM J. Control Optim., vol. 46, no. 2, pp. 496-540, 2007, doi: $10.1137 / 060658242$. 